\title{
Pathology of fatty liver disease
}

\author{
Elizabeth M Brunt \\ Department of Pathology, Saint Louis University School of Medicine, St Louis, MO, USA
}

\begin{abstract}
Fatty liver disease is currently recognized as a common cause of liver test elevation, paralleling the worldwide 'epidemic' of obesity in adults and children. In many clinical practices, there is recognition that liver biopsy evaluation is the only means of diagnosis (or exclusion) of fatty liver disease, as neither laboratory tests nor imaging studies to date can provide complete data related to amount of steatosis, inflammation, liver cell injury, fibrosis, and architectural remodeling. Liver biopsy evaluation also provides a means of 'grading and staging' the lesions of fatty liver disease and of detecting clinically unsuspected processes. Liver biopsy evaluation is often the primary end point in clinical trials of treatment, thus, standardization of diagnosis and methods of grading and staging have become important. In this review, these concepts as well as the pathophysiologic bases for them are discussed.

Modern Pathology (2007) 20, S40-S48. doi:10.1038/modpathol.3800680
\end{abstract}

Keywords: fatty liver disease; insulin resistance; grading; staging

The normal adult human liver may have up to $5 \%$ of its mass as lipid. The size of triglyceride droplets (macrovesicular or microvesicular) may be a clue to the underlying cause of the accumulation. When discussing 'fatty liver disease', most often we are referring to diseases that are characterized by predominantly large droplet steatosis (macrovesicular steatosis), or mixed large and small droplet steatosis. Small droplets that do not fill the entire hepatocyte are usually included in the macrovesicular category. Several primary liver diseases, such as hepatitis $\mathrm{C}$ and Wilson disease, hepatocellular adenoma and carcinoma, and certain drugs and toxins, such as steroids and alcohol, commonly show these forms of steatosis (Figure 1a). In contrast, 'true' microvesicular steatosis consists of much smaller, uniform fat droplets dispersed throughout the hepatocyte, and often requires special stains such as oil red $\mathrm{O}$ to detect (Figure 1b). This latter form is a manifestation of severe, recent liver injury, such as in Reye's syndrome and acute fatty liver of pregnancy and other processes of deranged mitochondrial $\beta$ oxidation.

Correspondence: Dr EM Brunt, MD, Department of Pathology, Saint Louis University School of Medicine, 4th Floor, FDT 3635 Vista Avenue, St Louis, MO 63110, USA.

E-mail: bruntem@slu.edu

Received 14 July 2006; accepted 26 July 2006

\section{Nonalcoholic fatty liver disease (NAFLD)}

\section{Background}

The significance of excess fat accumulation in the liver was recognized by pathologists in the 19th century. Associations of hepatic steatosis and cirrohosis with obesity, diabetes and alcohol have been documented in numerous large studies of American and European pathologists in the 20th century. Although not the first to use the term, credit is due to the study from the Mayo Clinic published in 1980 for establishing the moniker 'NASH' in the nomenclature of fatty liver disease. ${ }^{1}$ In this study, Ludwig et $a l^{1}$ carefully established the nonalcoholic nature of the 20 subjects with liver biopsies; $90 \%$ were obese, $65 \%$ were women, $25 \%$ were diabetic and/or hyperlipidemic, and $15 \%$ had hypertension. The subjects had been accrued based on a liver biopsy with features that had been characterized as 'alcohol-like'. Over the course of the next decade, the concept of NAFLD became accepted, in no small part due to pathologists' contributions in clinicopathologic studies. Today, we recognize that hepatic steatosis cannot be ignored as a mere histologic oddity, but rather is a significant finding or marker for potentially progressive liver disease. Most often, we see steatosis in the setting of alcohol-related liver disease, and hepatitis C (especially genotype 3 ), and in NAFLD and nonalcoholic steatohepatitis (NASH). In addition, in the setting of liver transplantation, large amounts of large droplet fat have been associated with initial poor function of the liver graft. 

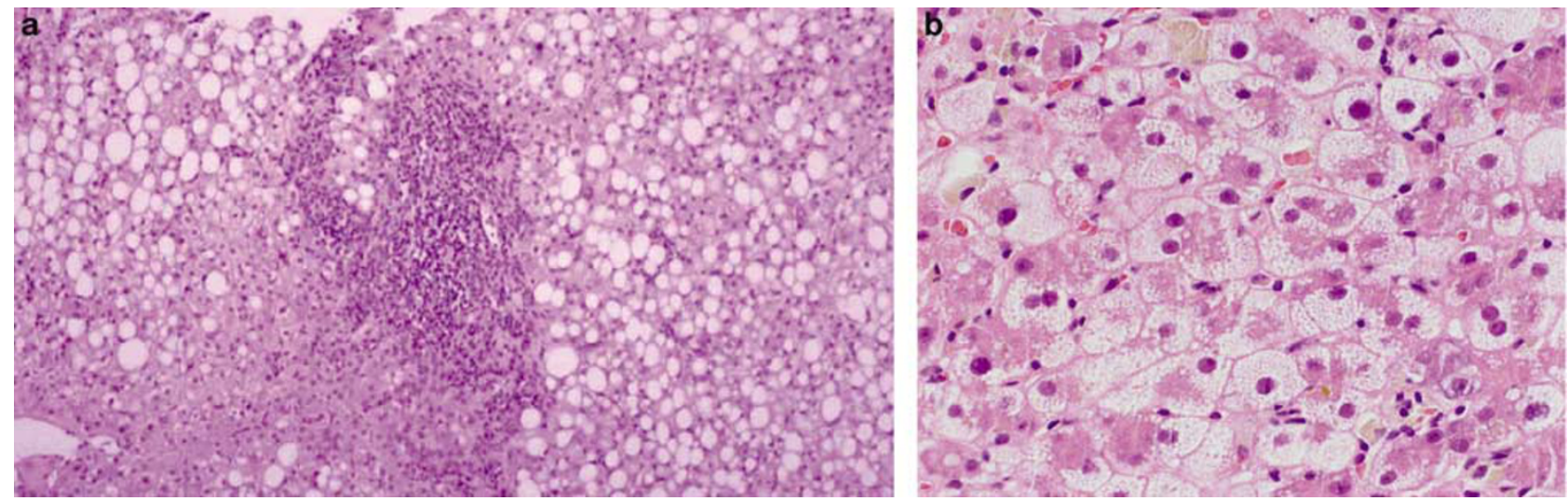

Figure 1 (a) Steatosis can be seen in varying amounts in a variety of liver diseases. The etiology of the steatosis may be due to a toxin, hepatitis C viral infection or host factors, but often cannot be determined by histologic evaluation. This is an example of steatosis, large and small droplet type, in hepatitis C in an overweight, diabetic individual. (b) Microvesicular steatosis is often initially considered as swollen, 'ballooned' hepatocytes. As shown in this example of acute fatty liver of pregnancy, the hepatocytes are enlarged and the cytoplasm is reticulated by the small aggregates of steatosis. Because of the significance of the clinical situations in which microvesicular steatosis is the dominant finding, stains on frozen sections are highly recommended for confirmation (photograph courtesy of Dr Linda Ferrell).

The epidemic of obesity throughout the world has brought awareness of NAFLD to the forefront of hepatology and medicine in the past quarter century. Currently, NAFLD is 'billed' as the most common form of chronic liver disease for adults in the United States and is growing to be such in Asia. The problem is now recognized in both adults and children. In all populations, obese and diabetic subjects are at higher risk than lean, nondiabetic individuals. It is unethical and impractical to perform population screening with liver biopsies, so several methods have been used to determine prevalence of NAFLD. Three cited studies are based on the data collected from the third National Health and Nutrition Evaluation Survey; ${ }^{2-4}$ all three studied ALT values in nonalcoholic subjects without serologic markers of liver disease; some additionally included values for $\mathrm{AST}^{2}$ and GGT, ${ }^{3}$ and only one specifically excluded diabetics. ${ }^{4}$ These studies found 'unexplained' elevated liver tests in US adults in $5.45,23$ and $2.8 \%$, respectively. The latter study showed that $68 \%$ of those could be accounted for by increased body mass index (BMI). ${ }^{4}$ An Italian population survey based on ultrasound evaluation of a cohort of 257 nondiabetic adults with no serologic evidence of liver disease showed evidence of steatosis ('bright liver') in $58 \% .^{5}$ This study also showed that the risk of steatosis was related to obesity and alcohol use. Another imaging study ${ }^{6}$ that measured hepatic triglyceride content in a multiethnic cohort of 2287 subjects in the US documented steatosis in $30 \%$ of subjects; interestingly, $79 \%$ of these subjects had normal ALT values. The ethnic distribution of steatosis reflected the well-documented ethnic distribution of NAFLDrelated cirrhosis: Hispanic $>$ Caucasian $>$ African American. A novel approach to detect prevalence has come from evaluation of newly diagnosed liver disease patients in a large clinic setting; ${ }^{7}$ in this study, $21 \%$ of 742 such patients were found to have NAFLD. Each of these types of studies have recognized drawbacks and liver tissue evaluation remains the 'gold standard' in the clinico-pathologic diagnosis of NAFLD for confirmation (or exclusion) of the diagnosis, for distinguishing NAFLD and $\mathrm{NASH}$, and to establish severity of inflammation and fibrosis. ${ }^{8}$

Studies have confirmed that routine clinical tests alone may misdiagnose (overdiagnose) NASH in a significant proportion of cases, ${ }^{9}$ and that not all 'unexplained' liver test abnormalities are the result of fatty liver disease. ${ }^{10}$ In Skelly et al's ${ }^{10}$ study, liver biopsy made a clinically significant and previously unsuspected diagnosis in $13 \%$ of cases, confirmed cryptogenic liver disease in $9 \%$ and documented normal liver in $6 \%$. Recent reports of liver disease in diabetics also confirm the value of tissue evaluation to identify entities other than fat, such as glycogenic hepatopathy, characterized by diffuse glycogenosis, ${ }^{11}$ and diabetic hepatosclerosis, which consists of dense perisinusoidal fibrosis and basement membrane deposition ${ }^{12}$ (Figure 2a and b). Neither of these entities is characterized by significant steatosis. These studies also emphasize the value of adequate clinical information, as both of these entities occur in the setting of insulin-dependent diabetes rather than obesity.

\section{Role of Liver Biopsy}

Histologic evaluation has also played an important role in broadening the concept of NAFLD from a liver disease of only obese individuals with elevated ALT, to an entity that can involve lean individuals, ${ }^{13,14}$ or subjects with normal ALT values as well. ${ }^{15}$ Most studies of NAFLD focus on subjects in whom other forms of liver disease have been 

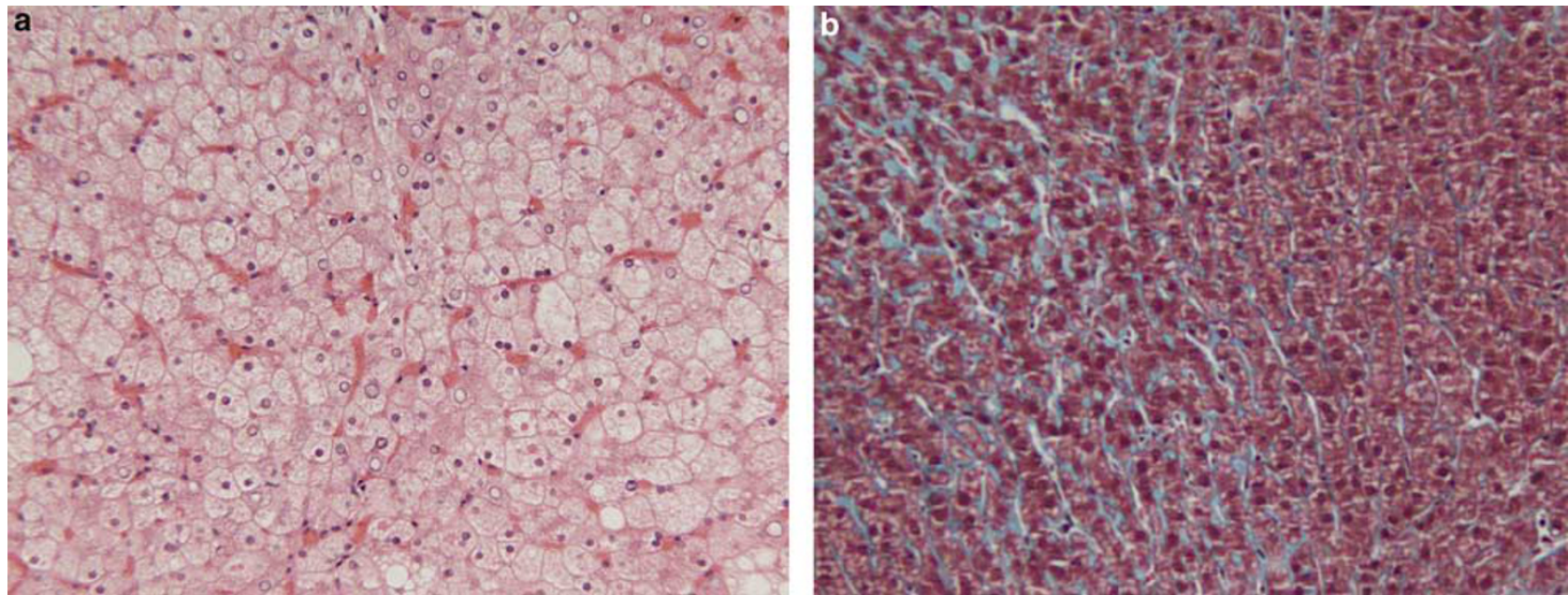

Figure 2 (a) Glycogenic hepatopathy. The hepatocyte cytoplasm is markedly expanded by excess glycogen, imparting a 'glassy' appearance. The parenchyma is diffusely involved. Small amounts of steatosis may also be present. (b) Diabetic hepatosclerosis, with patches of dense perisinusoidal fibrosis (trichrome stain).

excluded, but some have documented NAFLD/ NASH in subjects with concurrent chronic liver disease. ${ }^{16-18}$ A feature that often leads to diagnostic difficulties for clinicians is the presence of abnormal ANA, ASMA and AMA in the setting of NAFLD. Several studies to date that have shown from 3 to $40 \%$ of otherwise characteristic NAFLD/NASH subjects are positive for one of these antibodies, but the question of the significance of antibodies remains largely unanswered.

Liver pathology has also played a key role in the growing studies to evaluate clinical 'markers' of disease, such as adiponectin levels ${ }^{19}$ and the HAIR (hypertension, ALT, Insulin Resistance) score. ${ }^{20}$ Clinical studies to evaluate predictors of fibrosis, by definition, have relied on liver biopsy evaluation. ${ }^{21,22}$ Differing results in these studies may be because of some degree of differing histologic criteria and methods utilized for the evaluation of fibrosis evaluation. Biopsy studies have estimated the rate of progression of fibrosis in NAFLD from 0.08 stages/year ${ }^{23}$ to 0.28 stages/year. ${ }^{24}$ It is recognized that cirrhosis occurs in $19-33 \%$ of subjects with documented NASH; ${ }^{25}$ a recent study showed significantly increased rates of cirrhosis and mortality in 132 subjects with NAFLD and diabetes followed for 10 years compared with nondiabetic subjects with NAFLD. ${ }^{26}$

Experienced pathologists recognize that sample size, technique of obtaining the biopsy and processing are all important considerations in liver biopsies. Documentation of differences in grading and staging in chronic hepatitis in relationship to biopsy length have been reviewed, and it has been proposed that a $2 \mathrm{~cm}$ or more core with at least 11 complete portal tracts is probably necessary in order to grade and stage a case reliably. ${ }^{27}$ In NAFLD, this topic is also of great interest as the biopsy evaluation is such an integral component of the diagnosis. Differences in the lesions within the parenchyma, and thus in 'grade' and 'stage' have been shown in recent studies. ${ }^{28,29}$ As in all liver diseases, the type of liver biopsy (for instance, wedge biopsy, intraoperative biopsy, etc) available for evaluation and preparation of the tissue are significant considerations. Wedge biopsies may overestimate fibrosis if only parenchyma immediately adjacent to the liver capsule is over-represented. Intra-operative biopsies carried out after significant amounts of time under anesthesia have a risk of 'surgical hepatitis', which is characterized by clusters of polymorphonuclear leukocytes, often found around the terminal hepatic venules. When lobular inflammation is a key component of a score, as it is in NAFLD and NASH, these lesions can be troublesome. Finally, the use of the sponge in processing liver biopsies for histology is to be discouraged because of resultant mechanical artefacts including irregular indentations and triangular-shaped holes in the liver biopsy.

\section{Use of 'Special Stains'}

Many liver pathology texts emphasize the value of considering a variety of stains as 'routine' and not 'special' for evaluation; these typically include hematoxylin and eosin, trichrome (or an equivalent) for fibrosis, reticulin (or an equivalent) for evaluation of architecture, periodic acid Schiff with diastase for evaluation of globules, basement membranes and vessel walls, and an iron stain. Most find the trichrome particularly helpful to identify the early lesions of pericentral and perisinusoidal fibrosis seen in NASH. The other stains help to exclude other diagnostic possibilities. Globules of $\alpha$-1-antitrypsin and small amounts of either hepatocellular iron or reticulo-endothelial iron may be missed without the use of the periodic acid Schiff with diastase and iron stains, respectively. Iron stain is also useful in distinguishing iron from 
lipochrome pigment, copper and bile. Stains for copper or copper-associated protein are also considered useful by some if there is any concern of chronic bile duct or cholestatic injury, or Wilson disease. The regular use of 'special stains' adds very little to overall cost of the liver biopsy as each is not expensive, and if requested at the time of initial processing, will not result in wasting tissue by having to resurface the block.

\section{Histologic Criteria for NAFLD/NASH}

Of equal significance for diagnosis and studies in NAFLD are histologic criteria. Results of a blinded study of biopsies with clinico-pathologic correlations highlights the value of utilizing careful criteria to distinguish NAFLD and NASH. ${ }^{30}$ Ludwig's original, often-quoted manuscript continues to serve as a benchmark reference for pathologists in this disease. ${ }^{1}$

A survey of an international group of hepatopathologists who have published in the field on their criteria for diagnosing $\mathrm{NASH}^{31}$ illustrates the differences in opinions that exist in the field today. However, a common theme from each is that the diagnosis relies on a combination of lesions, a pattern of injury as it were, rather than a particular single lesion. The lesions most often noted to be included were steatosis, hepatocyte ballooning, lobular inflammation and perisinusoidal fibrosis; zone 3 accentuation and Mallory's hyaline were also noted by some. Two studies of observer variability have now shown that experienced liver pathologists who agree in advance what to study, and what the lesions 'look like' can have good or excellent agreement on the lesions of interest in NAFLD: steatosis, ballooning, lobular inflammation and fibrosis $;{ }^{32,33}$ this is particularly true in adult biopsies compared with pediatric biopsies. Although the literature is not entirely concordant, it is likely that most would agree that the finding of fat alone, or fat and inflammation alone, with no evidence of ballooning would qualify for definitive NAFLD, but not for NASH. The additional finding of ballooning would strongly support the diagnosis of active NASH, whereas the presence of zone 3 perisinusoidal fibrosis would support a diagnosis of previous/remote NASH, or can be seen in ongoing NASH.

Clinico-pathologic and pathophysiology correlates. In considering the histologic findings of NAFLD, it is important to consider the both clinical context in which this liver disease exists and the pathophysiology of the process. NAFLD has come to be recognized as the 'hepatic manifestation of the metabolic syndrome, ${ }^{14}$ from several studies documenting the close associations of the histologic findings with critical clinical features ${ }^{34,35}$ that have come to be grouped together because of a shared risk of increased cardiovascular disease. The 'metabolic syndrome' shares a common link of insulin resistance ${ }^{36}$ is variably defined and includes central (truncal) adiposity, abnormal glucose tolerance or diabetes, dyslipidemia and systemic hypertension. ${ }^{37}$ The 'two-hit' hypothesis of Day and James, ${ }^{38}$ although continually modified, remains a central paradigm in thinking of the complex pathogenesis of NAFLD/NASH. Briefly, insulin resistance and hyperinsulinemia (in combination with other factors including low adiponectin levels and elevated $\mathrm{TNF} \alpha$ ) result in and perpetuate hepatic steatosis; the fatty liver is considered more vulnerable to injury from a 'second hit(s)', and self-perpetuating cycles of cell injury and fibrogenic stimuli are initiated. There may be other 'hits' that lead to fibrosis and cirrhosis, and possibly, ultimately to hepatocellular carcinoma. Several likely gene candidates are actively being studied ${ }^{39}$ for each of the putative steps of the process; at least 23 have been shown to be involved in NAFLD ${ }^{40}$ and hundreds more are under investigation in fat metabolism and insulin interactions. ${ }^{36}$

The lesions of NAFLD/NASH can be understood in terms of the recognized and putative pathophysiology: ${ }^{39}$ the imbalance of free fatty acid delivery to the liver with overfeeding and insulin resistance relative to export and oxidation results in steatosis; reactive oxygen species, by-products of microsomal and peroxisomal oxidation, result in mitochondrial damage, decreased ATP, increased apoptosis, lipid peroxidation and cytokine release. Investigators have noted specific lesions in association with each of these steps. One of the first patterns recognizable in prefibrotic NASH in adults is the predominance of steatosis and injury in acinar zone 3; two groups have shown zonal localization of DNA damage, products of oxidative damage ${ }^{41}$ and expression of CYP $2 \mathrm{E} 1^{42}$ in zone 3 . Steatosis, while predominantly macrovesicular, may be mixed large and small droplet steatosis. Liver cell injury is most often noted in the form of ballooning with cell swelling; this was the key histologic feature that differentiated progressive NAFLD (Types 3 and 4) with an increased risk of cirrhosis and liver-related death in the study by Matteoni et al. ${ }^{43}$ Lobular inflammation is typically present, but mild and commonly mixed in nature. Portal inflammation is typically absent or mild, and when significant, it has been recommended that co-existent liver disease be considered. ${ }^{44}$ Increased portal inflammation has also recently been identified as a feature of resolution from the results of a recent treatment trial. ${ }^{45}$ Other features that may be present in NAFLD/NASH include Mallory's hyaline, which can be detected by immunohistochemical stains against ubiquitin and p62, acidophil bodies, megamitochondria and glycogenated nuclei. Hepatocellular and sinusoidal lining cell iron are rarely discussed except in focused studies of iron and NAFLD, but may be present if carefully examined. Fibrosis is initially present in acinar zone 3 in a perisinusoidal, 

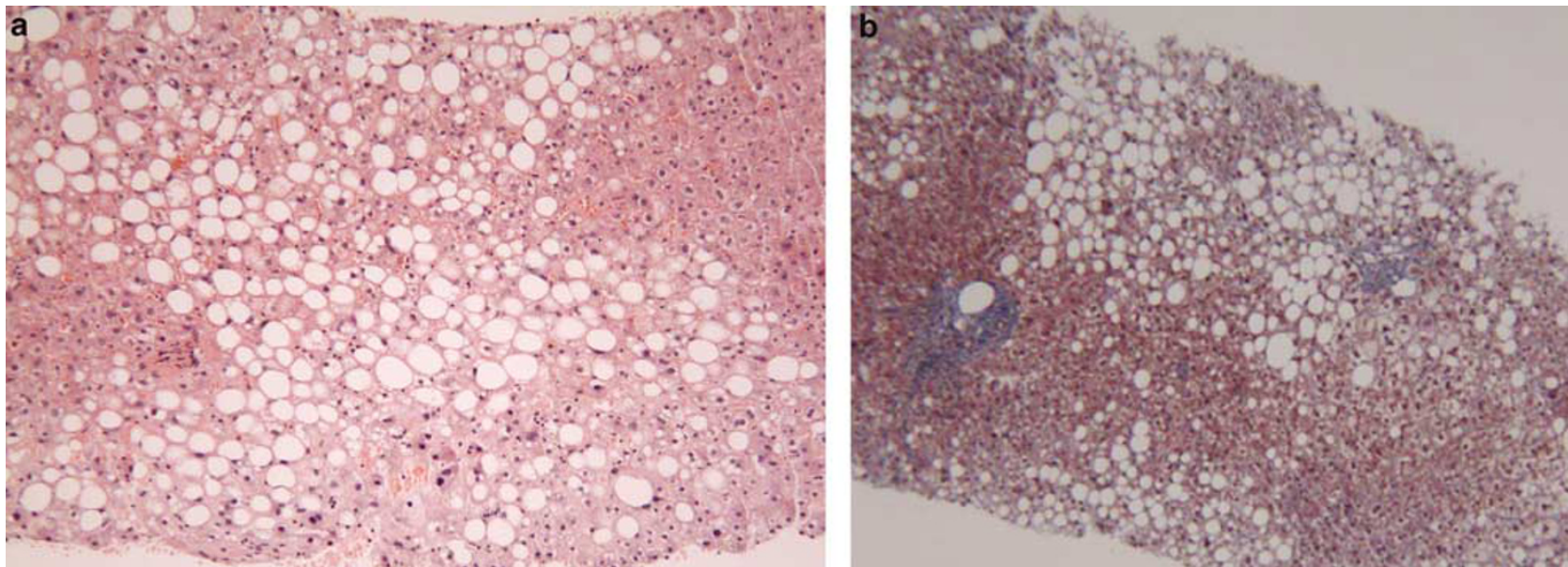

Figure 3 (a) Nonalcoholic steatohepatitis is characterized by zone 3 accentuation of the lesions of steatosis (large and small droplet type), ballooning and lobular inflammation. There may also be a component of portal chronic inflammation (not illustrated). The findings in this biopsy are indistinguishable from alcoholic steatohepatitis. (b) The earliest stage of fibrosis in NASH is zone 3 perisinusoidal collagen deposition in a 'chickenwire' pattern (central zone on right, portal tract on left). This pattern is also seen in alcoholic liver disease, and therefore is not useful in distinguishing the two (trichrome stain).

'chickenwire' pattern with sparing of the portal and periportal areas. Two studies utilizing evaluation of stellate cell activation by immunohistochemistry noted zone 3 accentuation in human liver biopsies of NASH. ${ }^{46,47}$ With progression, portal and periportal fibrosis may occur, as well as various forms of bridging fibrosis. Cirrhosis is a known complication of NASH. The physiologically altered liver with cirrhosis may or may not retain any or all of the active lesions of NASH; hence, many cases of 'cryptogenic' cirrhosis may have developed from 'burned-out' NASH. ${ }^{48}$ What is not known is in whom and how to accurately predict histological progression of fibrosis, that is, 'natural history' of NAFLD (Figure 3a and b); however, new concepts in the pathogenesis of fibrosis have been suggested. Very recently, a study in hepatitis $\mathrm{C}^{49}$ has been broadened to $\mathrm{NASH}^{50}$ to introduce the idea that the portal fibrosis of chronic liver disease correlates with the periportal ductular reaction, which may be 'driven' by hepatocyte proliferative arrest as measured by senescence markers. The putative cause(s) of hepatocyte proliferative arrest are speculated to be viral infection, steatosis, insulin resistance and increased body mass.

\section{Grading and Staging}

The concept of semiquantitative 'grading' and 'staging' the necroinflammatory lesions, fibrosis and architectural alterations in chronic hepatitis is well established. Batts and Ludwig ${ }^{51}$ describe the portal-based nature of these lesions for viral, autoimmune and some forms of metabolic chronic liver disease. Clearly, the lesions of NASH are different from those of portal-based injury of chronic hepatitis. Thus, a system for grading and staging NASH was proposed in $1999^{52}$ based on a blinded review of
52 biopsies from 51 clinical cases of NASH that recognized the constellation of lesions of NASH (Table 1a and 1b). Steatosis was present by definition and did not necessarily affect the grade, although greater amounts were typically present in more severe cases. The lesions that correlated with a 'gestalt' grade of mild, moderate and severe steatohepatitis were ballooning, lobular and portal inflammation. Fibrosis was evaluated for two components: zone 3 perisinusoidal fibrosis and portal-based fibrosis. It was noted that some cases had only the former, while cases with any portalbased fibrosis also had the former, or had bridging fibrosis, hence the staging system as proposed.

In 2002, the NIDDK sponsored NASH Clinical Research Network sponsored the Pathology Committee to develop a scoring system that would encompass the entire spectrum of NAFLD and provide a feature-based scoring system for the entire spectrum of NAFLD that could be applied to treatment trials in adults and children (Table 2a and $2 \mathrm{~b}$ ). The system was developed and validated by the nine pathologists of the group after two blinded readings of 32 adult biopsies and one blinded reading of 18 pediatric biopsies. ${ }^{33}$ This is a scoring system in which the components, steatosis, lobular inflammation and hepatocellular ballooning, are each semiquantitated, then added together for an aggregate activity score. The fibrosis score is a modification of the 'Brunt' fibrosis score above with additional subclassifications of stage 1 to account for delicate (1a) or dense (1b) perisinusoidal fibrosis and stage 1c to include 'portal only' fibrosis when noted. Biopsies with NAFLD Activity Scores (NAS) above 5 were nearly all correlated with a histologic diagnosis of 'definite NASH', whereas those of 0-2 were all diagnosed as 'definitely not NASH'; this was true in both adults and pediatric biopsies. A word of caution was noted: '...the primary purpose 
Table 1a NASH activity grade

\begin{tabular}{|c|c|c|c|}
\hline Grade & Steatosis & Ballooning & Inflammation \\
\hline $\begin{array}{l}\text { Mild, } \\
\text { Grade } 1\end{array}$ & $1-2$ (up to $66 \%$ ) & Minimal & $\begin{array}{l}\text { L: } 1-2 \\
\text { P: none-mild }\end{array}$ \\
\hline $\begin{array}{l}\text { Moderate, } \\
\text { Grade } 2\end{array}$ & $\begin{array}{l}2-3(>33 \% \\
\text { may be }>66 \%)\end{array}$ & Present & $\begin{array}{l}\text { L: } 2 \\
\text { P: mild- } \\
\text { moderate }\end{array}$ \\
\hline $\begin{array}{l}\text { Severe, } \\
\text { Grade } 3\end{array}$ & 3 & Marked & $\begin{array}{l}\text { L: } 3 \\
\text { P: mild- } \\
\text { moderate }\end{array}$ \\
\hline
\end{tabular}

Steatosis Grade 1: $\leq 33 \%$; Grade 2: $>33 \%<66 \%$; Grade 3: $\geq 66 \%$. $\mathrm{L}=$ lobular, $\mathrm{P}=$ portal.

Adapted from Brunt et al. ${ }^{52}$

Table 1b Staging of fibrosis for NASH

\begin{tabular}{ll}
\hline Stage & Histologic description \\
\hline 0 & No fibrosis \\
1 & Zone 3 perisinusoidal fibrosis only \\
2 & Zone 3 plus portal/periportal fibrosis \\
3 & As above with bridging fibrosis \\
4 & Cirrhosis
\end{tabular}

Adapted from Brunt et al. ${ }^{52}$

of the NAS is to assess overall histological change; it is not intended that numeric values replace the pathologist's diagnostic determination of steatohepatitis'.33

A third scoring system was published in $2005^{53}$ based on a review of 25 biopsies of predominantly Hispanic women by two blinded pathologists. The system is a multistep process of deriving an activity score (that is derived from adding lobular inflammation and necrosis plus Mallory bodies, plus hepatocyte ballooning, plus perisinusoidal fibrosis) to portal fibrosis scores to determine grades from 1 to 3 . The authors showed good $\kappa$ scores between the two pathologists and strong correlations and relevant clinical tests.

\section{NAFLD and Alcoholic Liver Disease (ALD)}

It is often stated in the literature that NASH is histologically 'identical' to ASH, but is that really true? There are several lesions of alcoholic liver disease that, to date, are not known in NAFLD, ${ }^{54}$ including sclerosing hyaline necrosis, the venoocclusive lesion first described by Goodman and Ishak, ${ }^{55}$ and alcoholic foamy degeneration. On the other hand, there are several biopsies of fatty liver disease for which the pathologist cannot be sure of the true etiology of liver disease, and cases in which obesity, diabetes and alcohol are all likely contributing factors (Figure 4). In addition, most of the standard literature in liver pathology notes that Mallory hyaline and neutrophilic infiltrates may be more prominent in ALD.
Table 2a Grading for NAFLD

\begin{tabular}{llll}
\hline NAS & Steatosis $^{\mathrm{b}}$ & Ballooning & Inflammation, lobular \\
\hline 0 & $<5 \%(0)$ & None (0) & None (0) \\
3 & $5-33 \%(1)$ & Rare or few (1) & $1-2$ foci per $20 \times$ field (1) \\
6 & $34-66 \%(2)$ & Many (2) & $2-4$ foci/ $20 \times$ field (2) \\
8 & $>66 \%(3)$ & Many (2) & $>4$ foci $/ 20 \times$ field (3) \\
\hline
\end{tabular}

NAS: NAFLD activity score.

${ }^{\mathrm{a}}$ The number in parentheses is the NAS score for each histologic feature. This is an example of how the composite NAS is derived for any case of NAFLD; in any case, the combinations of lesions may differ, and thus, the NAS would also.

${ }^{\mathrm{b}}$ Steatosis estimated by percent of fat in hepatocytes by examination at $4 \times$ and $10 \times$.

${ }^{\mathrm{C}}$ Includes all types of inflammatory cells in clusters in lobule (mononuclears, neutrophils, eosinophils).

Table 2b Staging for NAFLD

\begin{tabular}{ll}
\hline Stage & Histologic description \\
\hline 0 & No fibrosis \\
1a & Zone 3 perisinusoidal fibrosis, requires trichrome \\
& stain to identify \\
1b & Zone 3 perisinusoidal fibrosis, seen easily on H\&E \\
$1 \mathrm{c}$ & Periportal/portal fibrosis only \\
2 & Zone 3 plus portal/periportal fibrosis \\
3 & As above with bridging fibrosis \\
4 & Cirrhosis
\end{tabular}

Adapted from Kleiner et al. ${ }^{33}$

\section{Resolution of NAFLD}

Recent clinical treatment trials are affording us opportunities we had not realized in the past, as most are now being done with biopsies before and after intervention. One of the most interesting findings to date is the fact that some patients can have 'spontaneous' resolution of the lesions of NASH without specific clinical intervention, other than, perhaps, increased awareness of having a serious liver disease. This has been shown so far in three studies. ${ }^{45,56,59}$ Other findings that have been documented in clinical trials are shown in Table 3. Most ${ }^{45,56-58}$ studies, but not all ${ }^{59}$ have shown complete resolution of the features of NASH with treatment; some have shown new findings, such as a shift in inflammation that favors increased portal inflammation $^{45}$ or no change in portal inflammation. ${ }^{56}$ Fibrosis score decreased ${ }^{57}$ or the nature of fibrosis changed. ${ }^{45}$ Notable differences in these studies are the numbers of patients and the length of time of observation.

\section{Pediatric NAFLD}

Pediatric obesity and NAFLD are problems of growing significance in our country and throughout the world; the lesions of pediatric NAFLD, in many cases, are different than in adult cases; a recent 
study has classified the lesions into types 1 and 2 based on similarities, or lack thereof, with adult NAFLD. ${ }^{60}$ The major differences with adult NAFLD are greater steatosis, little or no hepatocellular ballooning or Mallory's hyaline, and portal accentuation over zone 3 accentuation in pediatric cases. This is a fledgling field that will continue to grow in the future.

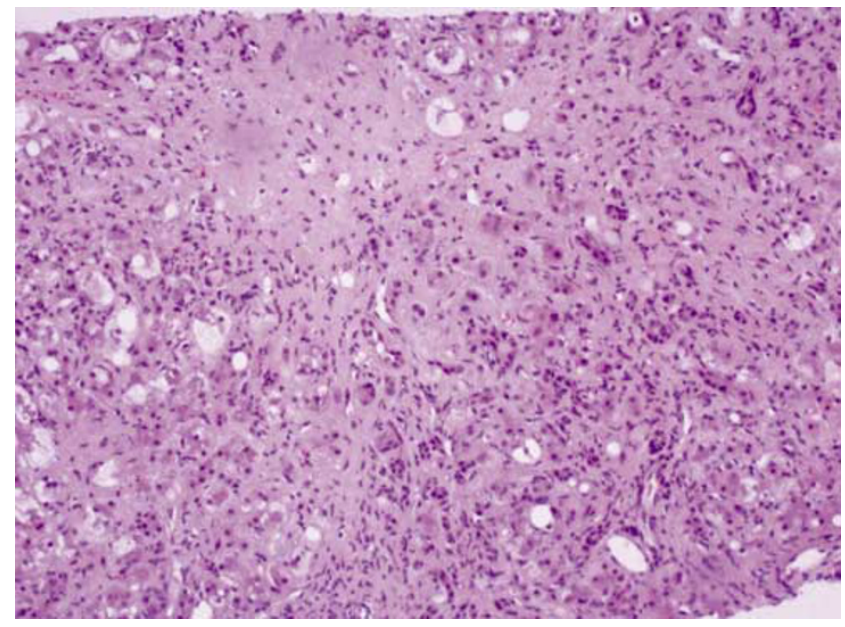

Figure 4 Sclerosing hyaline necrosis. The terminal hepatic venule is obliterated (upper left-center) and only visualized on special stains, which may highlight the remnants of the vein wall. Many of the surrounding hepatocytes have undergone necrosis, and several of the remaining hepatocytes are ballooned and/or have Mallory's hyaline with surrounding neutrophils, a lesion known as satellitosis.

\section{Differential Diagnoses}

Finally, we all are faced with biopsies from patients with clinically phenotypic NAFLD, but without fatty liver disease or even steatosis in the biopsy. Several questions arise in such biopsies. Do the lesions of NAFLD wax and wane? Do all the lesions of NAFLD/NASH (necessarily) 'disappear' with progression to cirrhosis or regression or do some features, such as fibrosis, remain? Further, is it possible that other causes of liver abnormalities can be detected by biopsy? Examples of cases that are seen in my experience include the following: (1) 'nonspecific' findings with evidence of prior hepatocellular injury; (2) globules of A1AT in unsuspected A1AT deficiency and (3) evidence of chronic cholestasis and, rarely, a lesion suggestive of PSC. The pathologist may also need to consider the possibility of autoimmune liver disease, iron overload or even Wilson disease.

Finally, even though the concept of the 'Metabolic Syndrome' is being questioned about its role in cardiovascular risk, ${ }^{61}$ this cluster of clinical findings currently retains a significant role in NAFLD. Perhaps with what we are learning in pathology, it is time for us to begin to broaden our 'spectrum' of pathology to include not only steatosis and steatohepatitis with cirrhosis but also the minimal lesions (or 'normal') and hepatocellular carcinoma in the setting of both cirrhotic and noncirrhotic liver.

Table 3 Histologic findings of recent clinical trials

Recent treatment trials: primary histologic findings

\begin{tabular}{lllll}
\hline Study & $(n)$ & Tx & Post-tx bx $(n)$ & Histologic findings reported \\
\hline $\begin{array}{l}\text { Neuschwander- } \\
\text { Tetri } \text { et } a l^{45}\end{array}$ & 30 overweight & Rosi & $\begin{array}{l}22 \\
48 \text { weeks }\end{array}$ & $\begin{array}{l}\text { 45\% no longer NASH; improved grade, steatosis, inflammation, } \\
\text { ballooning }(P=0.004)\end{array}$ \\
& & & $\begin{array}{l}\text { Shift toward increased portal CI }(P=0.02) \\
\text { No change in fibrosis score, shift from dense to delicate } \\
\text { perisinusoidal fibrosis }(P=0.02)\end{array}$
\end{tabular}

\begin{tabular}{|c|c|c|c|c|}
\hline Promrat et a ${ }^{56}$ & $\begin{array}{l}18 \text { overweight, } \\
\text { nondiabetic }\end{array}$ & Pio & $\begin{array}{l}18 \\
48 \text { weeks }\end{array}$ & $\begin{array}{l}67 \% \text { had histologic response; decreased steatosis, ballooning, } \\
\text { lobular inflammation, Mallory's hyaline, fibrosis }(P<0.05) \\
\text { No change in portal inflammation }\end{array}$ \\
\hline Lindor et $a l^{59}$ & 166 multicenter & $\begin{array}{l}\text { Urso v } \\
\text { Placebo }\end{array}$ & $\begin{array}{l}107 \\
104 \text { weeks }\end{array}$ & $\begin{array}{l}\text { Both groups had decreased steatosis, and no significant change } \\
\text { in inflammation or fibrosis } \\
\text { No statistical differences between groups }\end{array}$ \\
\hline Dixon et $a l^{57}$ & $\begin{array}{l}36 \text { morbid } \\
\text { obesity; } 12 \\
\text { steatosis only }\end{array}$ & LAGB & $\begin{array}{l}36 \\
36-204 \text { weeks }\end{array}$ & $\begin{array}{l}83 \% \text { no longer NASH; significant improvement in steatosis, } \\
\text { inflammation, fibrosis }(P<0.001) \text {. No change in portal } \\
\text { inflammation } \\
\text { Fibrosis: stage } \geq 2: 78 \% \rightarrow 13 \%(P<0.001)\end{array}$ \\
\hline Huang et $a l^{58}$ & 23 overweight & $\begin{array}{l}\text { Diet to } \\
\text { reduce } \\
\text { IR }\end{array}$ & $\begin{array}{l}15 \\
48 \text { weeks }\end{array}$ & $\begin{array}{l}60 \% \text { had histologic response; compared with nonresponders, } \\
\text { significant improvement in steatosis }(P=0.003) \text {, total score } \\
(P<0.0001)\end{array}$ \\
\hline
\end{tabular}

Tx, therapy; bx, biopsy; n, number; Rosi, rosiglitazone; Pio, pioglitazone; Urso, ursodeoxycholic acid; LAGB, laparoscopic adjustable gastric binding; IR, insulin resistance; CI, chronic inflammation. 


\section{References}

1 Ludwig J, Viggiano TR, McGill DB, et al. Nonalcoholic steatohepatitis: Mayo Clinic experiences with a hitherto unnamed disease. Mayo Clinic Proc 1980;55:434438.

2 Clark JM, Brancati FL, Diehl AM. The prevalence and etiology of elevated aminotransferase levels in the United States. Am J Gastroenterol 2003;98:960-967.

3 Clark JM, Brancati FL, Diehl AM. Nonalcoholic fatty liver disease. Gastroenterology 2002;122:1649-1657.

4 Ruhl CE, Everhart JE. Determinants of the association of overweight with elevated serum alanine aminotrasferase activity in the United States. Gastroenterolgy 2003;124:71-79.

5 Bellentani S, Sacciccio G, Masutti F, et al. Prevalence of and risk factors for hepatic steatosis in northern Italy. AnnInternal Med 2000;132:112-117.

6 Browning JD, Kumar KS, Saboorian H, et al. Ethnic differences in the prevalence of cryptogenic cirrhosis. Am J Gastroenterol 2004;99:292-298.

7 Weston SR, Leyden W, Murphy R, et al. Racial and ethnic distribution of nonalcoholic fatty liver in persons with newly diagnosed chronic liver disease. Hepatology 2005;41:372-379.

8 Neuschwander-Tetri BA, Caldwell SH. Nonalcoholic steatohepatitis: summary of an AASLD Single Topic Conference. Hepatology 2003;37:1202-1219.

9 Sorbi D, McGill DB, Thistle JL, et al. An assessment of the role of liver biopsies in asymptomatic patients with chronic liver test abnormalities. Am J Gastroenterol 2000;95:3206-3210.

10 Skelly MM, James PD, Ryder SD. Findings on liver biopsy to investigate abnormal liver function tests in the absence of diagnostic serology. J Hepatol 2001;35:195-199.

11 Chen YY, Brunt EM, Jakate SM, et al. Glycogenic hepatopathy: an under-recognized complication of diabetes mellitus. Mod Pathol 2004;17:297A.

12 Harrison SA, Brunt EM, Goodman ZD, et al. Diabetic hepatosclerosis: perisinusoidal hepatic fibrosis without steatohepatitis among diabetics. Hepatology 2003;38:266A.

13 Bacon BR, Farahvash MJ, Janney CG, et al. Nonalcoholic steatohepatitis-an expanded clinical entity. Gastroenterology 1994;107:1103-1109.

14 Marchesini G, Bugianesi E, Forlani G, et al. Nonalcoholic fatty liver, steatohepatitis, and the metabolic syndrome. Hepatology 2003;37:917-923.

15 Mofrad P, Contos MJ, Haque M, et al. Clinical and histologic spectrum of nonalcoholic fatty liver disease associated with normal ALT values. Hepatology 2003;37:1286-1292.

16 Brunt EM, Ramrakhiani S, Cordes BG, et al. Concurrence of histologic features of steatohepatitis with other forms of chronic liver disease. Mod Pathol 2003;16:49-56.

17 Sanyal AJ, Contos MJ, Sterling RK, et al. Nonalcoholic fatty liver disease in patients with hepatitis $C$ is associated with features of the metabolic syndrome. Am J Gastroenterol 2003;98:2064-2071.

18 Ong JP, Younossi ZM, Speer C, et al. Chronic hepatitis $\mathrm{C}$ and superimposed nonalcoholic fatty liver disease. Liver 2001;21:266-271.

19 Hui JM, Hodge A, Farrell GC, et al. Beyond insulin resistance in NASH: TNF-a or adiponectin? Hepatology 2004;40:46-54.
20 Dixon JB, Bhatal PS, O’Brien PE. Nonalcoholic fatty liver disease: predictors of nonalcoholic steatohepatitis and liver fibrosis in the severely obese. Gastroenterology 2001;121:91-100.

21 Ratziu V, Giral P, Charlotte F, et al. Liver fibrosis in overweight patients. Gastroenterology 2000;118:11171123.

22 Angulo P, Keach JC, Batts KP, et al. Independent predictors of liver fibrosis in patients with nonalcoholic steatohepatitis. Hepatology 1999;30:1356-1362.

23 Evans CDI, Oien KA, MacSween RNM, et al. Nonalcoholic steatohepatitis: a common cause of advanced liver injury? J Clin Pathol 2002;55:689-692.

24 Fassio E, Alvarez E, Dominguez N, et al. Natural history of nonalcoholic steatohepatitis: a longitudinal study of repeat liver biopsies. Hepatology 2004;40:820-826.

25 McCullough AJ. Update on nonalcoholic fatty liver disease. J Clin Gastroenterol 2002;34:255-262.

26 Younossi ZM, Gramlich T, Matteoni CA, et al. Nonalcoholic fatty liver disease in patients with type 2 diabetes. Clin Gastroenterol Hepatol 2004;2:262-265.

27 Guido M, Rugge M. Liver biopsy sampling in chronic viral hepatitis. Semin Liver Dis 2004;24:89-97.

28 Ratziu V, Charlotte F, Heurtier A, et al. Sampling variability of liver biopsy in nonalcoholic fatty liver disease. Gastroenterology 2005;128:1898-1906.

29 Goldstein NS, Hastah F, Galan MV, et al. Fibrosis heterogeneity in nonalcoholic steatohepatitis and hepatitis $\mathrm{C}$ virus needle core biopsy specimens. Am J Clin Pathol 2005;123:382-387.

30 Brunt EM, Neuschwander-Tetri BA, Oliver DA, et al. Nonalcoholic steatohepatitis: histologic features and clinical correlations with 30 blinded biopsies. Hum Pathol 2004;35:1070-1082.

31 Brunt EM. Nonalcoholic steatohepatitis. Semin Liver Dis 2004;24:3-20.

32 Younossi ZM, Gramlich T, Liu YC, et al. Nonalcoholic fatty liver disease-assessment of variability in pathologic interpretations. Mod Pathol 1998;11:560-565.

33 Kleiner DE, Brunt EM, Van Natta M, et al. Design and validation of a histological scoring system for nonalcoholic fatty liver disease. Hepatology 2005;41:13131321.

34 Pagano G, Pacini G, Musso G, et al. Nonalcoholic steatohepatitis, insulin resistance, and metabolic syndrome: further evidence for an etiologic association. Hepatology 2002;35:367-372.

35 Chitturi S, Abeygunasekera S, Farrell GC, et al. NASH and insulin resistance: insulin hypersecretion and specific association with insulin resistance syndrome. Hepatology 2002;35:373-379.

36 Bugianesi E, McCullough AJ, Marchesini G. Insulin resistance: a metabolic pathway to chronic liver disease. Hepatology 2005;42:987-1000.

37 Panel. Executive summary of the third report of the national cholesterol education program (NCEP) expert panel on detection, evaluation, and treatment of high blood cholesterol in adults (Adult Treatment Panel III). JAMA 2001;285:2486-2497.

38 Day CP, James OF. Steatohepatitis: a tale of two 'hits'? Gastroenterology 1998;114:842-845.

39 Day CP. Pathogenesis of steatohepatitis. Best Practice Res Clin Gastroenterol 2002;16:663-678.

40 Sreekumar R, Rosado B, Rasmussen D, et al. Hepatic gene expression in histologically progressive nonalcoholic steatohepatitis. Hepatology 2003;38:244-251. 
41 Seki S, Kitada T, Yamada T, et al. In situ detection of lipid peroxidation and oxidative DNA damage in nonalcoholic fatty liver diseases. J Hepatol 2002;37:56-62.

42 Farrell GC. Nonalcoholic steatohepatitis: what is it, and why is it important in the Asia-Pacific region? J Gastroenterol Hepatol 2003;18:124-138.

43 Matteoni CA, Younossi ZM, Gramlich T, et al. Nonalcoholic fatty liver disease: a spectrum of clinical and pathological severity. Gastroenterology 1999;116:1413-1419.

44 Clouston AD, Powell EE. Interaction of non-alcoholic fatty liver disease with other liver diseases. Best Practice Res Clin Gastroenterol 2002;16:767-781.

45 Neuschwander-Tetri BA, Brunt EM, Wehmeier KR, et al. Improvement in nonoalcoholic steatohepatitis following 48 weeks of treatment with the PPAR-g ligand rosiglitazone. Hepatology 2003;38:1008-1017.

46 Washington K, Wright K, Shyr Y, et al. Hepatic stellate cell activation in nonalcoholic steatohepatitis and fatty liver. Hum Pathol 2000;31:822-828.

47 Cortez-Pinto H, Baptista A, Camilo ME, et al. Hepatic stellate cell activation occurs in nonalcoholic steatohepatitis. Hepato-Gastroenterol 2001;48:87-90.

48 Caldwell SH, Oelsner DH, Iezzoni JC, et al. Cryptogenic cirrhosis: clinical characterization and risk factors for underlying disease. Hepatology 1999;29:664-669.

49 Powell EE, Jonsson JR, Clouston AD. Steatosis: co-factor in other liver diseases. Hepatology 2005;42:5-13.

50 Richardson MM, Jonsson JR, Powell EE, et al. Hepatic progenitor cell expansion and a periportal ductular reaction in NAFLD-relationship with impaired hepatocyte proliferation and fibrosis, and reversal with treatment. Hepatology 2005;42(Suppl 1):611A.

51 Batts KP, Ludwig J. Chronic hepatitis. An update on terminology and reporting. Am J Surg Pathol 1995;19:1409-1417.
52 Brunt EM, Janney CG, Di Bisceglie AM, et al. Nonalcoholic steatohepatitis: a proposal for grading and staging the histological lesions. Am J Gastroenterol 1999;94:2467-2474.

53 Mendler MH, Kamel G, Govindarajan S. Proposal for a histological scoring and grading system for nonalcoholic fatty liver disease. Liver Int 2005;25:294304.

54 Brunt EM. Alcoholic and nonalcoholic steatohepatitis. Clin Liver Dis 2002;6:399-420.

55 Goodman ZD, Ishak KG. Occlusive venous lesions in alcoholic liver disease. Gastroenterology 1985;83:786796.

56 Promrat K, Lutchman G, Uwaifo GI, et al. A pilot study of pioglitazone treatment for nonalcoholic steatohepatitis. Hepatology 2004;39:188-196.

57 Dixon JB, Bhatal PS, Hughes NR, et al. Nonalcoholic fatty liver disease: improvement in liver histological analysis with weight loss. Hepatology 2004;39:16471654.

58 Huang MA, Greenson JK, Chao C, et al. One-year intense nutritional counseling results in histological improvement in patients with nonalcoholic steatohepatitis: a pilot study. Am J Gastroenterol 2005;100:1072-1081.

59 Lindor KD, Kowdley KV, Heathcote EJ, et al. Ursodeoxycholic acid for treatment of nonalcoholic steatohepatitis: results of a randomized trial. Hepatology 2004;39:770-778.

60 Schwimmer JB, Behling C, Newbury R, et al. Histopathology of pediatric nonalcoholic fatty liver disease. Hepatology 2005;42:641-649.

61 Kahn R, Buse J, Ferrannini E, et al. The metabolic syndrome: time for a critical appraisal. Joint Statement from the American Diabetes Association and the European Association for the Study of Diabetes. Diabetologia 2005;48:1684-1699. 